\title{
Avaliação de jogos educativos no ensino de conteúdos acadêmicos: Uma revisão sistemática da literatura
}

\section{RESUMO}

Este estudo teve como objetivo fazer uma revisão sistemática da literatura de estudos empíricos que avaliaram o efeito de jogos educativos na aprendizagem de assuntos acadêmicos, comparando os efeitos de estudos com diferentes delineamentos de pesquisa. Na revisão, foram incluídos artigos empíricos e relatos de casos publicados em inglês ou português, de janeiro de 2006 a setembro de 2019, que mencionavam ou continham palavras-chave definidas previamente que avaliavam os jogos como método de intervenção para o ensino de conteúdo escolar formal. Inicialmente, 171 registros foram localizados e 24 foram selecionados com base nos critérios de inclusão. Verificou-se que: as crianças foram os principais participantes; os jogos eletrônicos foram os mais utilizados; e a Matemática foi a disciplina utilizada na maioria estudos. Em 13 estudos, o desenho do grupo foi empregado e verificou-se que o grupo experimental apresentou melhor desempenho acadêmico quando comparado ao grupo-controle. Em todos os 11 estudos que utilizaram um delineamento de caso único, o desempenho pós-teste foi maior que o do pré-teste. Esses resultados mostraram que os jogos tiveram o efeito de aumentar o desempenho acadêmico dos alunos.

Palavras-chave: Jogos educativos; Ensino-aprendizagem; Educação baseada em evidências.

\section{INTRODUÇÃO}

Assim como qualquer intervenção, a metodologia de ensino é crucial para a eficácia da aprendizagem. Uma das maneiras de os educadores terem acesso a práticas baseadas em evidências que avaliaram a utilidade de diferentes estratégias de ensino é por meio de revisões sistemáticas da literatura, uma vez que estas podem oferecer aos professores a possibilidade de conhecer os resultados de um conjunto amplo de pesquisas científicas que avaliaram determinado recurso, como os jogos.

Apesar de não haver consenso na literatura sobre a definição de jogos, há um acordo sobre suas características definidoras, como as regras, o objetivo a ser atingido no final, a participação voluntária e o sistema de feedback
Myenne Mieko Ayres Tsutsumi $^{i}$

Universidade Estadual de Londrina, Brasil

Paulo Roney Kilpp Goulart ii Universidade Federal do Pará, Brasil

Mauro Dias Silva Júnior ${ }^{i i i}$

Universidade de Brasília, Brasil

Verônica Bender Haydu iv Universidade Estadual de Londrina, Brasil

Érika Larissa de Oliveira Jimenéz ${ }^{\mathrm{v}}$

Universidade Federal do Pará, Brasil 
que informa ao jogador seu desempenho no jogo (Huizinga, 2000; McGonigal, 2012; Morford et al., 2014). No entanto, tais características não seriam suficientes para o ato de ensinar. Segundo De Rose e Gil (2003), o jogo é um espaço em que há regras que especificam quais comportamentos levam a quais consequências e, com isso, seria possível determinar quais comportamentos devem ser ensinados mesmo que o jogo usado não tenha sido construído para ser educativo. É necessário o arranjo adequado das condições educacionais para que o ensino possa ser eficaz (Gris et al., 2018; Perkoski \& Souza, 2015).

Considerando as características dos jogos citadas anteriormente, 0 jogador decide participar sem que seja coagido a isso. Ele precisa seguir regras para atingir o objetivo final e recebe feedback do quão próximo está de alcançá-lo. Esse protagonismo que o jogador exerce nessa atividade tem sido explorado como uma forma alternativa ao ensino tradicional e é denominado (e.g., Allen et al., 2011; Groccia \& Buskist, 2011; Michaelsen \& Sweet, 2011) metodologias ativas de ensino. Segundo Prince (2004), a definição de metodologias ativas não é consensual, mas é possível traçar alguns aspectos que caracterizam essa prática. Basicamente, ao usar metodologias ativas como a aprendizagem ativa, o professor tem como objetivo criar condições para que os alunos desenvolvam autonomia na produção do conhecimento. 0 professor organiza questões, projetos ou problemas que deverão ser solucionados por meio da colaboração entre pares, pesquisas individuais e compartilhamento das informações. 0 papel do professor passa a ser o de guia da trajetória do aluno na busca de fontes confiáveis de informação, ensino da leitura crítica do material, propiciar feedbacks sobre as respostas dadas pelos alunos, além de promover discussões que irão aprofundar o estudo do tema (Prince, 2004). Algumas dessas características das metodologias ativas podem ser encontradas em jogos, nos quais os participantes são protagonistas da história, buscam outras fontes de informação para solucionar os problemas, colaboram com outros jogadores e constroem conhecimento acerca da atividade que o jogo propõe.

Assim como a concepção sobre jogos pode variar, sua utilização dentro do contexto de ensino pode assumir diferentes funções (ver Perkoski \& Souza, 2015). A Base Nacional Comum Curricular (Ministério da Educação, 2015), por exemplo, considera que jogos e brincadeiras nas aulas de Educação Física possuem valor em si mesmos (i.e., são atividades importantes para a promoção da saúde e do entretenimento) e não necessariamente são usados como meios para ensinar um assunto específico apesar de inseridos no contexto escolar.

Um levantamento prévio verificou que há um número considerável de publicações que relataram que o emprego de jogos, no contexto de ensino, é um método eficaz (por exemplo, Hirsh-Pasek et al., 2008; Kishimoto et al., 2011; Neves et al., 2015). Segundo a pesquisa conduzida por Bennett et al. (2001), os professores entrevistados acreditavam que o brincar era uma condição ideal para que as crianças pudessem aprender. Além de melhorar a qualidade da aprendizagem, pois se trata de um contexto natural, espontâneo em que a criança pode ser "ela mesma" e permite a exploração e experimentação do ambiente sem medos e restrições. Vale destacar que os termos jogo e brincadeira não são sinônimos, mas serão tratados aqui como conceitos 
correspondentes. É importante ressaltar que jogos e brincadeira são termos com conceitos diferentes. Os jogos são ambientes planejados para promover a ocorrência de determinados comportamentos que reúnem elementos fundamentais para uma programação de ensino adequada, principalmente no que se refere à disposição imediata de consequências para os citados comportamentos, o que pode ser utilizado no contexto educacional.

Os benefícios do uso de jogos para o ensino de diversas habilidades acadêmicas também são demonstrados a partir dos resultados de pesquisas científicas que avaliam a efetividade dos próprios jogos. Por exemplo, uma revisão sistemática realizada por Jabbar e Felicia (2015) visou avaliar como o engajamento é caracterizado nos jogos educativos e como isso afeta a aprendizagem. Os autores identificaram, nos estudos analisados, que os jogos proveem oportunidades de engajamento dos jogadores e que a aprendizagem no jogo tem relação com as estruturas e características dos jogos. Clark et al. (2016) revisaram sistematicamente pesquisas sobre "jogos digitais e aprendizagem" para estudantes que completaram o ensino médio. Eles sintetizaram os estudos que fizeram em comparações entre jogos e outras mídias, assim como em comparações entre o design dos jogos de realidade aumentada e jogos-padrão que utilizam tecnologia equivalente. Os autores verificaram que houve diferenças nos aspectos motivacionais, de ética e consciência de trabalho, e auto-avaliação positiva, além das melhorias gerais no desempenho intrapessoal para o grupo que foi exposto às condições dos jogos. Ke (2016) fez uma revisão sobre jogos digitais educacionais com o objetivo de sintetizar as heurísticas do design de jogos desse tipo considerando a integração que existe de forma intrínseca na jogabilidade no que diz respeito à aprendizagem voltada para um domínio específico. Os autores levantaram cinco temas que compõem uma potencial estrutura de design de jogo e que integram a relação aprender-brincar em jogos digitais educacionais. A literatura consultada para a revisão indicou que os jogos são projetados para abarcar conhecimentos prévios dos jogadores, bem como para promover conhecimentos novos. Além disso, eles encontraram que os domínios específicos da aprendizagem são integrados no desenvolvimento dos jogos utilizando conceitos como imersão, interatividade, experiências orientadas para a descoberta, e que a prática e exploração são mais prevalentes do que o conhecimento relacionado a compreensão e organização. Essas revisões exemplificam a necessidade da operacionalização dos elementos dos jogos que visam integrar a aprendizagem, a interação e a participação ativa do jogador, algo que colabora com o planjeamento de atividades educativas diferentes do ensino tradicional.

O contato com a literatura que coleciona pesquisas empíricas e teóricas sobre o uso de determinadas metodologias e o registro dos procedimentos executados durante as aulas e seus efeitos na aprendizagem dos alunos pode ajudar o professor a planejar suas aulas. Com isso, esse planejamento pode ser realizado de maneira mais bem fundamentada e com maiores chances de êxito, além de melhor adequá-lo às necessidades dos alunos, conforme apontaram Blumberg (2011) e Silva (2014). Esses autores destacam que a prática educativa pode-se tornar limitada, imprecisa, incompatível com a realidade de sala de aula e insensível às reais necessidades dos alunos. 
A ideia principal desse tipo de educação é usar o procedimento científico para o planejamento das aulas e utilizar o conhecimento resultante nas decisões educacionais (Blumberg, 2011; Silva, 2014; Thomas \& Pring, 2007). Com base em produções científicas, o professor estaria constantemente engajado em investigar e questionar suas práticas, podendo adequá-las conforme os resultados.

As revisões sistemáticas acerca de jogos encontradas na busca feita para o presente estudo avaliaram diferentes aspectos sobre os efeitos de jogos; no entanto, não foram encontradas revisões que respondessem à seguinte questão: a utilização de jogos é uma metodologia de ensino eficaz? Esta questão é o problema de pesquisa do presente estudo, que se caracteriza como um mapeamento da literatura e que teve como objetivo realizar uma revisão sistemática da literatura de estudos empíricos que avaliaram o efeito de jogos educativos sobre a aprendizagem de matérias e/ou conteúdos acadêmicos, analisando comparativamente os efeitos de estudos com diferentes delineamentos de pesquisa.

\section{MÉTODO}

\subsection{CRITÉRIOS DE ELEGIBILIDADE}

0 presente estudo consiste em uma revisão sistemática baseada no protocolo PRISMA (Liberati et al., 2009), o qual utiliza cinco componentes para a realização do processo de revisão identificados como PICOS - Participants, Interventions, Comparators, Outcomes; em português: Participantes, Intervenções, Comparadores, Resultados. Foram selecionados estudos empíricos com base nos seguintes critérios de inclusão: (a) Participantes de qualquer faixa etária; (b) Intervenções - estudos que avaliaram o efeito de jogos educativos; (c) Comparadores - delineamento de grupos (grupo experimental e grupo controle) e delineamento intra-sujeito; (d) Resultados - contendo o resultado da comparação entre os grupos e/ou entre pré e pósteste. Ainda como critérios foram especificados o período de publicação dos artigos - janeiro de 2006 a setembro de 2019 - e artigos publicados em inglês ou português. Além disso, somente foram considerados para a seleção aqueles artigos que estivessem disponíveis no Portal de Periódicos da CAPES ou na Internet de forma gratuita.

\subsection{FONTES DE INFORMAÇÃO E BUSCA}

As buscas foram feitas nas base de dados SciELO e Web of Science. Na base de dados Web of Science, a busca foi feita na aba de pesquisa básica, preenchendo o espaço destinado para busca de palavras-chave e escolhendo o campo "Tópico", o qual faz buscas nas seguintes seções de um texto: título, resumo, palavras-chave do autor e palavras adicionais (tradução livre do termo original em inglês Keywords Plus). Foram utilizados três desses espaços visto que, para o presente estudo, três palavras-chave foram escolhidas, combinadas e agrupadas por meio do operador booleano AND (ver Tabela 1). Para criar os outros espaços de busca, clicava-se em "+ Adicionar linha". 
Para a base de dados SciELO, foi utilizada a plataforma scielo.org com acesso pelo Portal de Periódicos da CAPES, como supracitado. Nesta base de dados, a busca foi feita na opção "Pesquisa avançada" preenchendo o espaço destinado para busca de palavras-chave e escolhendo o campo "Todos os índices", que faz busca das seguintes informações: título, resumo, periódico, financiador, autor e ano de publicação. As mesmas combinações de palavras-chave utilizadas na busca feita na Web of Science foram utilizadas na SciELO. Também foi utilizado o operador booleano AND, e, para criar os outros espaços de busca, clicava-se em "Adicionar outro campo +". Para ambas as bases de dados, as palavras-chave poderiam ocorrer no título, no resumo e/ou no corpo do texto.

Tabela 1

Combinações das Palavras-Chave Utilizadas nas Bases de Dados Web of Science e SCIELO

\begin{tabular}{llll}
\hline 1 & game AND & "academic achievement" AND & "school learning" \\
\hline 2 & game AND & "academic achievement" AND & teaching \\
3 & game AND & "school learning" AND & teaching \\
4 & jogo AND & "rendimento escolar" AND & "aprendizagem escolar" \\
5 & jogo AND & "rendimento escolar" AND & ensino \\
6 & jogo AND & "aprendizagem escolar" AND & ensino \\
\hline
\end{tabular}

\subsection{SELEÇÃO DOS REGISTROS}

Etapa 1. Pesquisa nas bases de dados, selecionando os registros cujos títulos continham uma das combinações das palavras-chave previamente definidas e que estavam de acordo com os demais critérios de inclusão especificados anteriormente.

Etapa 2. Após isso, houve a leitura dos resumos por dois juízes, que trabalharam de forma independente. Eles consideravam aceito aquele resumo que contivesse respostas afirmativas para pelo menos três das seguintes perguntas: (a) É um artigo empírico e um relato de experiência/caso?; (b) Faz a utilização de jogo(s) como método de intervenção para o ensino de algum conteúdo formal escolar?; (c) Especifica e descreve o tipo de jogo usado?; (d) A coleta de dados ocorreu na escola e/ou na sala de aula?; (e) Informa qual a disciplina que foi ensinada por meio do jogo?; (f) Informa quais foram os resultados de aprendizagem encontrados?

Em caso de dúvidas quanto ao critério das três perguntas, os juízes aceitavam o resumo com restrições. Compuseram a amostra somente os registros que foram classificados como aceitos ou aceitos com restrição por ambos os juízes. Se um juiz avaliasse como aceito e o outro como aceito com restrições, o artigo era incluído para a próxima etapa. No caso de discordância entre os juízes, um terceiro juiz foi o responsável por avaliar a inclusão ou exclusão do registro em que houve a divergência. Nesta etapa, foi feito o cálculo 
de concordância de Kappa utilizando o pacote estatístico SPSS 18 para verificar a conformidade no julgamento dos artigos feitos pelos dois juízes.

Foram excluídos da seleção os artigos de estudos que investigaram o uso de elementos de jogos sem usar jogos propriamente ditos (e.g., gamificação) como intervenção, que não avaliaram o desempenho acadêmico dos participantes e que investigaram a utilização de jogos em aulas de Educação Física. Esse último critério é justificado pela consideração da Base Nacional Comum (Ministério da Educação, 2015) sobre jogos serem atividades importantes para a saúde e entretenimento e não necessariamente serem instrumento de ensino de conteúdo formal. Publicações editoriais (por exemplo, apresentação dos artigos de um determinado volume), revisões de livros, discussões teóricas, apresentações em conferências, saudações, entrevistas ou homenagens também foram excluídas da revisão.

\subsection{PROCESSO DE COLETA DE DADOS}

Os artigos referentes aos registros selecionados foram baixados da Internet $\mathrm{e}$ lidos integralmente. As informações eram coletadas com base nas seguintes questões, por um dos autores do presente estudo: (a) Quais as idades e a escolarização dos participantes do estudo?; (b) Qual(is) tipo(s) de jogo (e.g., eletrônico ou outro tipo) é/são utilizado(s) como método de intervenção?; (c) Qual(is) disciplina(s) ou conteúdo(s) é/são ensinado(s)?; (d) Qual(is) o(s) delineamento(s) utilizado(s)?; (e) Qual(is) o(s) instrumento(s) de avaliação do desempenho utilizado(s)?; (f) Qual(is) o(s) tratamento(s) estatístico(s) que foi/foram empregado(s)?. Foram excluídos os artigos que não apresentavam informações para as seis questões.

\section{RESULTADOS}

Foram encontrados 171 registros na base de dados Web of Science e nenhum na base de dados SciELO. Os títulos e resumos dos 171 registros foram lidos e julgados por dois juízes. Foi encontrado um grau de concordância entre juízes de $\mathrm{K}=0.44$ (<.001). Por não se enquadrarem nos critérios de inclusão, 87 artigos foram excluídos. Ao total, 84 registros foram selecionados para download, tendo sido recuperados 76 , que foram lidos integralmente e avaliados por um dos juízes, com base nas seis perguntas especificadas no método. No final, seguindo as três etapas de seleção de artigos, 24 artigos foram incluídos para a tabulação dos dados. A Figura 1 apresenta o fluxograma da seleção de registros e artigos, bem como seus resultados. 


\section{Figura 1.}

Fluxograma da pesquisa e número de artigos localizados, excluídos e recuperados.
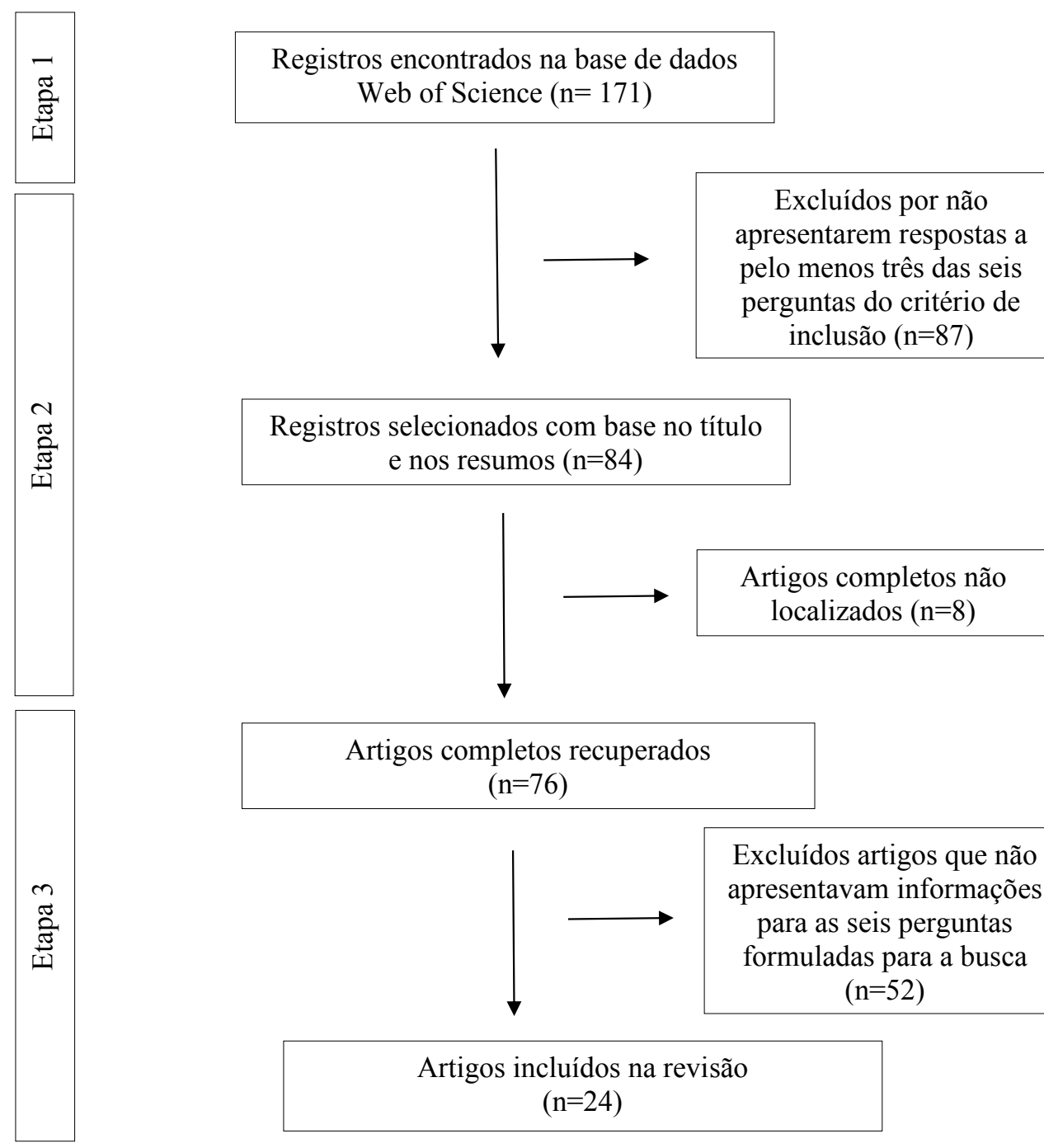

Registros selecionados com base no título e nos resumos $(n=84)$
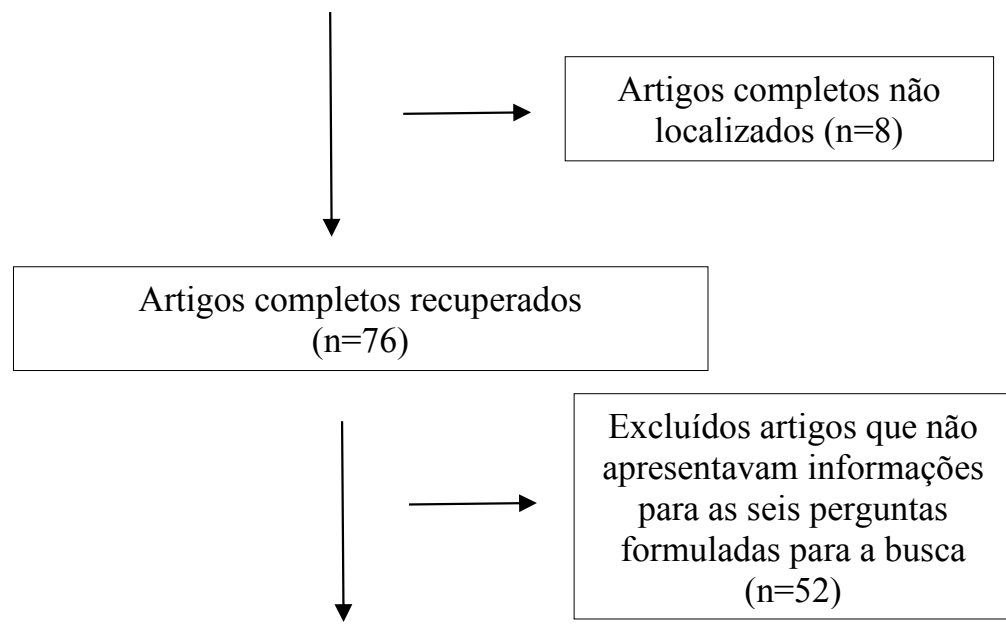

Artigos incluídos na revisão $(n=24)$

Os dados obtidos dos artigos estão dispostos na Tabela 2, a qual apresenta a caracterização geral dos estudo (como os autores e ano da publicação); a quantidade, a idade dos participantes e a sua escolarização (séries escolares do Ensino Fundamental, Ensino Médio ou Ensino Superior); o tipo de jogo utilizado; a disciplina/conteúdo que o jogo focalizava; os instrumentos utilizados para coletar informações sobre o desempenho escolar; os tipos de delineamento e de tratamento estatístico. 
Tabela 2

Caracterização Geral dos Estudos da Revisão de Literatura, com Identificação

dos Autores, Características dos Participantes, Tipo de Jogo Utilizado,

Disciplina Ensinada, Tipo de Instrumento de Avaliação do Desempenho e Tipo

de Tratamento Estatístico

\begin{tabular}{|c|c|c|c|c|c|c|c|}
\hline \multirow{2}{*}{$\begin{array}{l}\text { Autores } \\
\text { e ano da } \\
\text { publicação }\end{array}$} & \multicolumn{2}{|c|}{ Número de participantes } & \multirow{2}{*}{$\begin{array}{l}\text { Tipo de jogo } \\
\text { utilizado }\end{array}$} & \multirow{2}{*}{$\begin{array}{l}\text { Disciplina/ } \\
\text { conteúdo } \\
\text { ensinado }\end{array}$} & \multirow{2}{*}{$\begin{array}{l}\text { Delineamento } \\
\text { da pesquisa }\end{array}$} & \multirow{2}{*}{$\begin{array}{l}\text { Instrumento de } \\
\text { avaliação do } \\
\text { desempenho }\end{array}$} & \multirow{2}{*}{$\begin{array}{l}\text { Tratamento } \\
\text { estatístico }\end{array}$} \\
\hline & Idade & $\begin{array}{l}\text { Escolaridade/ } \\
\text { Série do Ens. } \\
\text { Fundamental }\end{array}$ & & & & & \\
\hline $\begin{array}{l}\text { Cahyana et } \\
\text { al., } 2017\end{array}$ & $\begin{array}{l}\text { Adolescentes } \\
\qquad(\mathrm{n}=40)\end{array}$ & Ensino Médio & $\begin{array}{l}\text { Jogo eletrônico } \\
\text { (computador) }\end{array}$ & Química & Grupo & $\begin{array}{c}\text { Avaliação de } \\
\text { desempenho elaborada } \\
\text { pelos autores }\end{array}$ & ANOVA \\
\hline $\begin{array}{l}\text { Cardozo et } \\
\text { al., } 2016\end{array}$ & $\begin{array}{l}\text { Adultos de } \\
18-25 \text { anos } \\
(n=62)\end{array}$ & $\begin{array}{l}\text { Ensino } \\
\text { Superior }\end{array}$ & $\begin{array}{l}\text { Jogo não } \\
\text { eletrônico } \\
\text { (quebra- } \\
\text { cabeça) }\end{array}$ & $\begin{array}{l}\text { Fisiologia } \\
\text { cardíaca }\end{array}$ & Grupo & $\begin{array}{c}\text { Avaliação de } \\
\text { desempenho elaborada } \\
\text { pelos autores }\end{array}$ & Teste $\mathrm{t}$ \\
\hline $\begin{array}{l}\text { Castro et al., } \\
2014\end{array}$ & $\begin{array}{l}\text { Crianças de } \\
\text { 7-10 anos } \\
(n=300)\end{array}$ & 2. ${ }^{a}$ série & $\begin{array}{l}\text { Jogo eletrônico } \\
\text { (computador) }\end{array}$ & Matemática & Grupo & $\begin{array}{c}\text { Teste aritmético } \\
\text { contido no Teste de } \\
\text { Desempenho Escolar } \\
\text { (TDE) }\end{array}$ & Teste $\mathrm{t}$ \\
\hline $\begin{array}{l}\text { Cardozo et } \\
\text { al., } 2016\end{array}$ & $\begin{array}{l}\text { Adultos } \\
(n=198)\end{array}$ & $\begin{array}{l}\text { Ensino } \\
\text { Superior }\end{array}$ & $\begin{array}{l}\text { Jogo não } \\
\text { eletrônico } \\
\text { (“Brainteaser } \\
\text { activity”) }\end{array}$ & Química & Grupo & $\begin{array}{l}\text { Attitude toward the } \\
\text { Subject of Chemistry } \\
\text { Inventory Version } 2 \\
\text { (ASClv2) }\end{array}$ & MANOVA \\
\hline $\begin{array}{l}\text { Heng et al., } \\
2015\end{array}$ & $\begin{array}{l}\text { Crianças } \\
(n=62)\end{array}$ & 7. ${ }^{a}$ série & $\begin{array}{l}\text { Jogo eletrônico } \\
\text { (computador) }\end{array}$ & Evolução & Caso único & $\begin{array}{c}\text { Avaliação de } \\
\text { desempenho elaborada } \\
\text { pelos autores }\end{array}$ & $\begin{array}{l}\text { ANOVA e } \\
\text { Correlação } \\
\text { de Pearson }\end{array}$ \\
\hline $\begin{array}{l}\text { El Kah \& } \\
\text { Lakhouaja, } \\
\quad 2018\end{array}$ & $\begin{array}{l}\text { Crianças } \\
(n=46)\end{array}$ & 2. ${ }^{a}$ série & $\begin{array}{l}\text { Jogo eletrônico } \\
\text { (computador) }\end{array}$ & $\begin{array}{l}\text { Leitura e } \\
\text { ortografia }\end{array}$ & Grupo & $\begin{array}{c}\text { Avaliação de } \\
\text { desempenho elaborada } \\
\text { pelos autores }\end{array}$ & Teste $\mathrm{t}$ \\
\hline $\begin{array}{l}\text { Fazio et al., } \\
2016\end{array}$ & $\begin{array}{l}\text { Crianças } \\
(n=26)\end{array}$ & 4. ${ }^{a}-5 .^{a}$ séries & $\begin{array}{l}\text { Jogo eletrônico } \\
\text { (computador) }\end{array}$ & Matemática & Caso único & $\begin{array}{c}\text { Avaliação de } \\
\text { desempenho elaborada } \\
\text { pelos autores }\end{array}$ & $\begin{array}{l}\text { Estatística } \\
\text { descritiva }\end{array}$ \\
\hline $\begin{array}{l}\text { Fotaris et al., } \\
2016\end{array}$ & $\begin{array}{l}\text { Adultos de } \\
19-25 \text { anos } \\
(n=106)\end{array}$ & $\begin{array}{l}\text { Ensino } \\
\text { Superior }\end{array}$ & $\begin{array}{l}\text { Jogo não } \\
\text { eletrônico } \\
\text { (“Quem } \\
\text { quer ser um } \\
\text { milionário”) }\end{array}$ & Programação & Grupo & $\begin{array}{l}\text { Avaliação da própria } \\
\text { escola }\end{array}$ & Não usou \\
\hline $\begin{array}{l}\text { Gabriel et al., } \\
2012\end{array}$ & $\begin{array}{l}\text { Crianças } \\
(n=269)\end{array}$ & 4. ${ }^{a}-5 .^{a}$ séries & Jogo de cartas & Matemática & Grupo & $\begin{array}{c}\text { Avaliação de } \\
\text { desempenho elaborada } \\
\text { pelos autores }\end{array}$ & ANCOVA \\
\hline $\begin{array}{l}\text { Johnson- } \\
\text { Glenberg \& } \\
\text { Hekler, } 2013\end{array}$ & $\begin{array}{c}\text { Crianças } \\
(n=19)\end{array}$ & 4. ${ }^{a}$ série & $\begin{array}{l}\text { Jogo eletrônico } \\
\text { (kinect/ } \\
\text { console) }\end{array}$ & Nutrição & Caso único & $\begin{array}{c}\text { Avaliação de } \\
\text { desempenho elaborada } \\
\text { pelos autores }\end{array}$ & Teste $\mathrm{t}$ \\
\hline
\end{tabular}




\begin{tabular}{|c|c|c|c|c|c|c|c|}
\hline $\begin{array}{l}\text { Johnson- } \\
\text { Glenberg et } \\
\text { al., } 2014\end{array}$ & $\begin{array}{c}\text { Crianças } \\
(n=20)\end{array}$ & 6. ${ }^{a}-7 .^{a}$ séries & $\begin{array}{l}\text { Jogo eletrônico } \\
\text { (kinect/ } \\
\text { console) }\end{array}$ & Nutrição & Grupo & $\begin{array}{c}\text { Avaliação de } \\
\text { desempenho elaborada } \\
\text { pelos autores }\end{array}$ & Teste $\mathrm{t}$ \\
\hline $\begin{array}{c}\text { Kanthan \& } \\
\text { Senger, } 2011\end{array}$ & $\begin{array}{l}\text { Adultos } \\
(n=114)\end{array}$ & $\begin{array}{l}\text { Ensino } \\
\text { Superior }\end{array}$ & $\begin{array}{l}\text { Jogo eletrônico } \\
\text { (computador) }\end{array}$ & $\begin{array}{l}\text { Patologias } \\
\text { humanas }\end{array}$ & Caso único & $\begin{array}{c}\text { Avaliação de } \\
\text { desempenho elaborada } \\
\text { pelos autores }\end{array}$ & Teste $\mathrm{t}$ \\
\hline Ke, 2008 & $\begin{array}{l}\text { Crianças de } \\
10-13 \text { anos } \\
(n=15)\end{array}$ & 4. ${ }^{a}-5 .^{a}$ séries & $\begin{array}{l}\text { Jogo eletrônico } \\
\text { (computador) }\end{array}$ & Matemática & Caso único & $\begin{array}{c}\text { Teste de matemática } \\
\text { (GSAT) }\end{array}$ & MANOVA \\
\hline $\begin{array}{l}\text { Klisch et al., } \\
2012\end{array}$ & $\begin{array}{l}\text { Adolescentes } \\
\qquad(\mathrm{n}=444)\end{array}$ & Ensino Médio & $\begin{array}{l}\text { Jogo eletrônico } \\
\text { (computador) }\end{array}$ & Química & Caso único & Avaliação da escola & Teste $\mathrm{t}$ \\
\hline $\begin{array}{l}\text { Kosmas et al., } \\
2018\end{array}$ & $\begin{array}{l}\text { Crianças } \\
\text { 7-10 anos } \\
(n=52)\end{array}$ & 2. ${ }^{a}-3 .^{a}$ séries & $\begin{array}{l}\text { Jogo eletrônico } \\
\text { (kinect/ } \\
\text { console) }\end{array}$ & Vocabulário & Caso único & $\begin{array}{l}\text { Versão grega do “Word } \\
\text { Finding Vocabulary } \\
\text { Test” de Renfrew (1998) }\end{array}$ & Teste $\mathrm{t}$ \\
\hline $\begin{array}{l}\text { McCarroll et } \\
\text { al., } 2009\end{array}$ & $\begin{array}{l}\text { Adultos com } \\
\text { média de } 22 \\
\text { anos }(n=40)\end{array}$ & $\begin{array}{l}\text { Ensino } \\
\text { Superior }\end{array}$ & $\begin{array}{l}\text { Jogo não } \\
\text { eletrônico } \\
\text { (“fast date”) }\end{array}$ & Kinesiologia & Grupo & Avaliação da escola & Teste $\mathrm{t}$ \\
\hline $\begin{array}{l}\text { Rico et al., } \\
2015\end{array}$ & $\begin{array}{l}\text { Adultos } \\
(\mathrm{n}=38)\end{array}$ & $\begin{array}{l}\text { Ensino } \\
\text { Superior }\end{array}$ & $\begin{array}{l}\text { Jogo eletrônico } \\
\text { (kinect/ } \\
\text { console) }\end{array}$ & $\begin{array}{l}\text { Língua } \\
\text { estrangeira } \\
\text { (inglês) }\end{array}$ & Caso único & $\begin{array}{l}\text { Teste elaborado } \\
\text { pelos instrutores de } \\
\text { Linguagens }\end{array}$ & $\begin{array}{l}\text { Estatística } \\
\text { descritiva }\end{array}$ \\
\hline $\begin{array}{l}\text { Roesch et al., } \\
2016\end{array}$ & $\begin{array}{c}\text { Crianças } \\
(n=47)\end{array}$ & 5. ${ }^{a}-6 .^{a}$ séries & $\begin{array}{l}\text { Jogo eletrônico } \\
\text { (computador) }\end{array}$ & $\begin{array}{l}\text { Ortografia e } \\
\text { Matemática }\end{array}$ & Caso único & $\begin{array}{l}\text { Avaliação elaborada } \\
\text { pelos autores }\end{array}$ & ANOVA \\
\hline $\begin{array}{c}\text { Stolaki \& } \\
\text { Economides, } \\
2018\end{array}$ & $\begin{array}{l}\text { Adultos } \\
(n=90)\end{array}$ & $\begin{array}{l}\text { Ensino } \\
\text { Superior }\end{array}$ & $\begin{array}{l}\text { Jogo eletrônico } \\
\text { (computador) }\end{array}$ & Criatividade & Caso único & $\begin{array}{c}\text { Avaliação da própria } \\
\text { escola }\end{array}$ & $\begin{array}{l}\text { ANOVA e } \\
\text { Teste } t\end{array}$ \\
\hline $\begin{array}{l}\text { Tüzün et al., } \\
2009\end{array}$ & $\begin{array}{c}\text { Crianças } \\
(n=13)\end{array}$ & 4. ${ }^{a}-5 .^{a}$ séries & $\begin{array}{l}\text { Jogo eletrônico } \\
\text { (computador) }\end{array}$ & Geografia & Caso único & $\begin{array}{c}\text { Avaliação de } \\
\text { desempenho elaborada } \\
\text { pelos autores }\end{array}$ & Teste $\mathrm{t}$ \\
\hline Yang, 2012 & $\begin{array}{l}\text { Adolescentes } \\
\text { de } 15-16 \\
\text { anos }(n=44)\end{array}$ & 9. ${ }^{a}$ série & $\begin{array}{l}\text { Jogo eletrônico } \\
\text { (computador) }\end{array}$ & $\begin{array}{l}\text { Civilidade e } \\
\text { sociedade }\end{array}$ & Grupo & $\begin{array}{c}\text { Avaliação de } \\
\text { desempenho elaborada } \\
\text { pelos autores }\end{array}$ & $\begin{array}{l}\text { ANOVA e } \\
\text { ANCOVA }\end{array}$ \\
\hline Yang, 2015 & $\begin{array}{l}\text { Adolescentes } \\
\text { de } 16-17 \\
\text { anos }(n=77)\end{array}$ & Ensino Médio & $\begin{array}{l}\text { Jogo eletrônico } \\
\text { (computador) }\end{array}$ & Administração & Grupo & $\begin{array}{l}\text { Pré-teste obtido pelas } \\
\text { notas dos estudantes } \\
\text { no semestre anterior; } \\
\text { e pós-teste elaborado } \\
\text { pelos autores }\end{array}$ & MANCOVA \\
\hline $\begin{array}{l}\text { Yen-Hua et } \\
\text { al., } 2012\end{array}$ & $\begin{array}{l}\text { Crianças de } \\
10-11 \text { anos } \\
\quad(n=83)\end{array}$ & 4. ${ }^{a}$ série & $\begin{array}{l}\text { Jogo eletrônico } \\
\text { (computador) }\end{array}$ & Matemática & Grupo & $\begin{array}{c}\text { Avaliação de } \\
\text { desempenho elaborada } \\
\text { pelos autores }\end{array}$ & ANOVA \\
\hline $\begin{array}{l}\text { Yien et al., } \\
2011\end{array}$ & $\begin{array}{c}\text { Crianças } \\
(n=66)\end{array}$ & 3. a série & $\begin{array}{l}\text { Jogo eletrônico } \\
\text { (computador) }\end{array}$ & Nutrição & Grupo & $\begin{array}{c}\text { Teste sobre } \\
\text { conhecimento em } \\
\text { nutrição elaborado por } \\
\text { Lo (2006) }\end{array}$ & ANCOVA \\
\hline
\end{tabular}

Como especificado na Tabela 2, a caracterização geral dos estudos mostrou que 13 estudos tiveram como participantes crianças com idades variando entre 7 e 13 anos; quatro estudos contaram com adolescentes variando entre 15 e 17 anos; e sete estudos com adultos sem idades especificadas que cursavam o Ensino Superior. 
Em 19 estudos foram usados jogos eletrônicos e em cinco foram usados jogos não eletrônicos. Além disso, a Matemática foi a disciplina/conteúdo mais focalizado nos jogos $(n=6)$. Em relação aos instrumentos utilizados para mensurar o desempenho escolar, em 13 estudos os próprios autores elaboraram instrumentos de avaliação; em quatro, os autores utilizaram as avaliações feitas pela instituição de ensino em que os participantes eram alunos; e em quatro, também, foram utilizados testes padronizados. Em um estudo para o pré-teste, os autores utilizaram a avaliação da escola. 0 pós-teste foi elaborado pelos autores.

Para avaliar se haveria efeitos do uso de jogos na aprendizagem/rendimento escolar dos alunos, os pesquisadores utilizaram dois tipos de delineamento metodológico (ver Tabela 2). No delineamento experimental com grupos ( $\mathrm{n}=13)$, dois grupos foram utilizados: um recebia a intervenção (grupo experimental) e outro não a recebia (grupo controle). Os desempenhos de ambos os grupos foram medidos antes e depois da intervenção no grupo experimental e comparados. No delineamento de caso único $(n=11)$, um único grupo recebeu a intervenção e os desempenhos antes e depois dela foram comparados.

Tabela 3

Comparação dos Resultados da Avaliação do Desempenho Acadêmico dos

Participantes de Cada Estudo de Acordo com o Delineamento de Pesquisa Utilizado

\begin{tabular}{|c|c|c|}
\hline Estudos & Delineamentos & $\begin{array}{l}\text { Comparação dos resultados } \\
\text { da avaliação do desempenho acadêmico }\end{array}$ \\
\hline Cahyana et al., 2017 & Grupo & $\mathrm{GE}>\mathrm{GC}$ \\
\hline Cardozo et al., 2016 & & $\mathrm{GE}=\mathrm{GC}$ \\
\hline Castro et al., 2014 & & $\mathrm{GE}>\mathrm{GC}$ \\
\hline Cha et al., 2017 & & $\mathrm{GE}>\mathrm{GC}$ \\
\hline El Kah \& Lakhouaja, 2018 & & $\mathrm{GE}>\mathrm{GC}$ \\
\hline Fotaris et al., 2016 & & $\mathrm{GE}>\mathrm{GC}$ \\
\hline Gabriel et al., 2012 & & $\mathrm{GE}>\mathrm{GC}$ \\
\hline Johnson-Glenberg et al., 2014 & & $\mathrm{GE}>\mathrm{GC}$ \\
\hline McCarroll et al., 2009 & & $\mathrm{GE}=\mathrm{GC}$ \\
\hline Yang, 2012 & & $\mathrm{GE}=\mathrm{GC}$ \\
\hline Yang, 2015 & & $\mathrm{GE}>\mathrm{GC}$ \\
\hline Yen-Hua et al., 2012 & & $\mathrm{GE}>\mathrm{GC}$ \\
\hline Yien et al., 2011 & & $\mathrm{GE}>\mathrm{GC}$ \\
\hline Cheng et al., 2015 & Caso único & Pós-teste > Pré-teste \\
\hline Fazio et al., 2016 & & Pós-teste > Pré-teste \\
\hline Johnson-Glenberg \& Hekler, 2013 & & Pós-teste > Pré-teste \\
\hline Ke, 2008 & & Pós-teste > Pré-teste \\
\hline Klisch et al., 2012 & & Pós-teste > Pré-teste \\
\hline Kosmas et al., 2018 & & Pós-teste > Pré-teste \\
\hline Rico et al., 2015 & & Pós-teste > Pré-teste \\
\hline Stolaki \& Economides, 2018 & & Pós-teste > Pré-teste \\
\hline Tüzün et al., 2009 & & Pós-teste > Pré-teste \\
\hline
\end{tabular}

Nota. GE = Grupo Experimental, GC = Grupo Controle; Os sinais de $>$ ou = indicam que o desempenho acadêmico do grupo ou fase da esquerda foi maior ou igual que o da direita. 
Nos 13 estudos onde foram utilizados delineamentos de grupo, o grupo experimental era exposto ao jogo e o grupo controle não, o qual permanecia assistindo às aulas sobre um determinado conteúdo no formato tradicional da escola. Em 10 desses estudos, os pesquisadores verificaram que, após a participação no jogo, a aprendizagem/rendimento acadêmico do grupo experimental foi superior à do grupo controle e, em três deles, não foram observadas diferenças entre os grupos. Em 11 estudos, os participantes foram avaliados como controles de si mesmos (delineamento de caso único) e, em todos esses estudos, o desempenho dos participantes no pós-teste foi superior ao do pré-teste (ver Tabela 3).

Do total de artigos incluídos nesta revisão, apenas um não utilizou tratamento estatístico. Dos 23, dois utilizaram estatística descritiva, dois utilizaram Análise Multivariada da Variância (MANOVA), um utilizou Análise Multivariada da Covariância (MANCOVA), seis utilizaram Análise de Variância (ANOVA), três utilizaram Análise de Covariância (ANCOVA) e 11 utilizaram o Teste $t$ de Student. Dos que utilizaram ANOVA, três deles usaram outros testes estatísticos em conjunto (e.g., correlação de Pearson, Teste t e Análise de Covariância). Dos que utilizaram ANCOVA, um usou outro teste combinado (ANOVA) e, dos que utilizaram Teste $t$, um usou outro teste (ANOVA).

\section{DISCUSSÃO}

Os resultados dos estudos avaliados nesta revisão, de modo geral, sugerem que o uso de jogos como intervenção pode ser um método eficaz para melhorar o desempenho acadêmico de alunos de diversas idades. Nos estudos que utilizaram delineamento de grupo, o desempenho acadêmico dos participantes do grupo experimental (i.e., aquele que foi exposto ao uso do jogo) foi superior ao do grupo controle (i.e., o que não utilizou jogo e continuou sendo exposto ao ensino convencional de sua escola). Esse resultado contribui para fundamentar as práticas já utilizadas de usar jogos como ferramentas de ensino (cf. Hirsh-Pasek et al., 2008; Kishimoto et al., 2011; Neves et al., 2015). Além disso, a maioria dos estudos da revisão mostrou que, independentemente do conteúdo, alunos expostos aos jogos apresentaram um melhor desempenho acadêmico quando comparados àqueles que continuaram no ensino tradicional. Nos estudos que utilizaram delineamento de caso único, todos os alunos apresentaram desempenho acadêmico superior no pós-teste quando comparados ao pré-teste. Isso indica que o jogo foi uma ferramenta que alterou o desempenho escolar.

A análise sobre o funcionamento dos jogos deve considerar suas principais características definidoras, como colocado por Jabbar e Felicia (2015): na atividade, o jogador é um agente ativo de suas ações. Esse protagonismo que o aluno adquire quando se torna jogador pode ser um aspecto fundamental para o ensino. De forma contrária ao que o ensino tradicional tem conduzido, o aluno-jogador tem um objetivo a cumprir e participa de forma voluntária, algo que já fornece informações sobre o seu nível de engajamento na atividade. Além disso, a programação do jogo conta com um sistema de feedback. Passo a passo, o comportamento do aluno-jogador é moldado e 
se torna pré-requisito para a aprendizagem de comportamentos complexos, como ler, escrever um texto autoral, pesquisar, colaborar, calcular, planejar (de Rose \& Gil, 2003).

Apesar de não ser um tema tão recente, o apelo por metodologias ativas de ensino tem tomado conta da discussão sobre as mudanças de que o ensino tradicional precisa. Diferentes métodos de ensino têm sido criados e testados a fim de reinventar a forma como os estudantes aprendem e os professores ensinam (Allen et al., 2011; Michaelsen \& Sweet, 2011).

A utilização dos delineamentos pode ser acompanhada de testes estatísticos. Foi o caso de 23 dos 24 estudos avaliados nesta revisão. 0 fato de os escores terem sido registrados por diferentes instrumentos pode ter sido o responsável pela escolha de diferentes tratamentos estatísticos.

Apesar de a maioria dos testes usados ter sido paramétrica, nem todos os estudos informaram se a amostra era normalmente distribuída, nem se os participantes foram randomicamente alocados para a formação dos grupos. Isso pode implicar limitações da generalização dos resultados, sendo a representatividade da população questionada, caso os autores não tenham considerado esses critérios.

A maior parte dos testes avaliava, por meio de comparações, as médias obtidas entre os grupos (e.g., ANOVA e Teste t). Três estudos conduziram uma análise de covariância (ANCOVA), a qual ocorre quando o pesquisador quer controlar o efeito de uma segunda variável (i.e., a covariável poderia ser a experiência prévia dos alunos com jogos e dispositivos eletrônicos), além da que ele está manipulando, sobre a variável que ele está medindo. Dessa forma, os pesquisadores podem obter um efeito da variável de interesse sobre a medida comportamental sem a interferência do efeito de uma ou mais covariáveis. Recomenda-se que o valor da significância de alfa (tradicionalmente estipulado em 0,05 ), além de mencionado, seja acompanhado de um teste de magnitude de efeito e do poder estatístico do teste. Estas medidas adicionais informam a magnitude de efeito na qual a medida comportamental é afetada pela variável manipulada e a sensibilidade do teste em detectar a existência ou não de uma diferença significativa.

Em conclusão, os artigos empíricos e estudos de caso selecionados nesta revisão mostraram que a faixa etária da população-alvo é variada, assim como a sua escolarização, e que diferentes disciplinas podem ser adaptadas para seu ensino via jogos, o que mostra que a utilização de jogos pode ser uma ferramenta versátil e útil para o ensino de diferentes disciplinas. Pesquisas futuras podem ampliar o número de bases de dados consultadas; os idiomas considerados foi algo que restringiu o alcance das conclusões aqui apresentadas. Futuros trabalhos poderão focar em um tipo específico de jogo. Podem se ampliar as fontes de buscas, os idiomas e as palavras-chave utilizadas para que a pesquisa por evidências ajude na tomada de decisão pedagógica. 


\section{REFERÊNCIAS}

Allen, D. E., Donham, R. S., \& Bernhardt, S. A. (2011). Problem-based learning. New Directions for Teaching and Learning, 2011(128), 21-29. https://doi. org/10.1002/tl.465

Bennett, N., Wood, L., \& Rogers, S. (2001). Teaching through play: Teachers' thinking and classroom practice (2nd ed.). Open University Press.

Blumberg, P. (2011). Making evidence-based practice an essential aspect of teaching. Journal of Faculty Development, 25(3), 27-32.

Cahyana, U., Paristiowati, M., Savitri, D. A., \& Hasyrin, S. N. (2017). Developing and application of mobile game based learning (M-GBL) for high school students performance in chemistry. Eurasia Journal of Mathematics, Science and Technology Education, 13(10), 7037-7047. https://doi.org/10.12973/ejmste/78728

Cardozo, L. T., Miranda, A. S., Moura, M. J. C. S., \& Marcondes, F. K. (2016). Effect of a puzzle on the process of students' learning about cardiac physiology. Advances in Physiology Education, 40(3), 425-431. https://doi.org/10.1152/ advan.00043.2016

Castro, M. V., Bissaco, M. A. S., Panccioni, B. M., Rodrigues, S. C. M., \& Domingues, A. M. (2014). Effect of a virtual environment on the development of mathematical skills in children with dyscalculia. PLoS ONE, 9(7), e103354. https://doi.org/10.1371/journal.pone.0103354

Cha, J., Kan, S.-Y., Wahab, N. H. A., Aziz, A. N., \& Chia, P. W. (2017). Incorporation of brainteaser game in basic organic chemistry course to enhance students' attitude and academic achievement. Journal of the Korean Chemical Society, 61(4), 218-222. https://doi.org/10.5012/jkcs.2017.61.4.218

Cheng, M. T., Lin, Y. W., \& She, H. C. (2015). Learning through playing Virtual Age: Exploring the interactions among student concept learning, gaming performance, in-game behaviors, and the use of in-game characters. Computers \& Education, 86, 18-29. https://doi.org/10.1016/j.compedu.2015.03.007

Clark, D. B., Tanner-Smith, E. E., \& Killingsworth, S. S. (2016). Digital games, design, and learning: A systematic review and meta-analysis. Review of Educational Research, 86(1), 79-122. https://doi.org/10.3102/0034654315582065

de Rose, J. C., \& Gil, M. S. C. A. (2003). Para uma análise do brincar e de sua função educacional - A função educacional do brincar. In M. Z. S. Brandão, F. C. S. Conte, F. S. Brandão, Y. K. Ingberman, C. B. Moura, V. M. Silva, \& S. M. Oliane (Eds.), Sobre comportamento e cognição (pp. 373-382). ESETec.

El Kah, A., \& Lakhouaja, A. (2018). Developing effective educative games for Arabic children primarily dyslexics. Education and Information Technologies, 23(6), 2911-2930. https://doi.org/10.1007/s10639-018-9750-2

Fazio, L. K., Kennedy, C. A., \& Siegler, R. S. (2016). Improving children's knowledge of fraction magnitudes. PLOS ONE, 11(10), 1-14. https://doi.org/10.1371/ journal.pone. 0165243

Fotaris, P., Mastoras, T., Leinfellner, R., \& Rosunally, Y. (2016). Climbing up the leaderboard: An empirical study of applying gamification techniques to a computer programming class. The Electronic Journal of E-Learning, 14(2), 94-110.

Gabriel, F., Coché, F., Szucs, D., Carette, V., Rey, B., \& Content, A. (2012). Developing children's understanding of fractions: An intervention study. Mind, Brain, and Education, 6(3), 137-146. https://doi.org/10.1111/j.1751-228X.2012.01149.x 
Gris, G., Perkoski, I. R., \& Souza, S. R. (2018). Jogos educativos: Aspectos teóricos, aplicações e panorama da produção nacional por analistas do comportamento. In D. L. O. V. Boas, F. Cassas, H. L. Gusso, \& P. C. M. Mayer (Eds.), Comportamento em foco: Ensino, comportamento verbal e análise conceitual (7. ${ }^{\mathrm{a}} \mathrm{ed} ., \mathrm{pp}$. 50-67). Associação Brasileira de Psicologia e Medicina Comportamental (ABPMC).

Groccia, J. E., \& Buskist, W. (2011). Need for evidence-based teaching. New Directions for Teaching and Learning, 2011(128), 5-11. https://doi.org/10.1002/tl.463

Hirsh-Pasek, K., Golinkoff, R. M., Berk, L. E., \& Singer, D. (2008). A mandate for playful learning in preschool: Applying the scientific evidence. Oxford University Press.

Huizinga, J. (2000). Homo ludens (4. ${ }^{a}$ ed.). Editora Perspectiva S.A.

Jabbar, A. I. A., \& Felicia, P. (2015). Gameplay engagement and learning in game-based learning: A systematic review. Review of Educational Research, 85(4), 1-40. https://doi.org/10.3102/0034654315577210

Johnson-Glenberg, M. C., \& Hekler, E. B. (2013). "Alien Health Game”: An embodied exergame to instruct in nutrition and MyPlate. Games for Health Journal, 2(6), 354-361. https://doi.org/10.1089/g4h.2013.0057

Johnson-Glenberg, M. C., Savio-Ramos, C., \& Henry, H. (2014). “Alien Health": A nutrition instruction exergame using the kinect sensor. Games for Health Journal, 3(4), 241-251. https://doi.org/10.1089/g4h.2013.0094

Kanthan, R., \& Senger, J. (2011). The impact of specially designed digital game-based learning in undergraduate pathology and medical education. Archives of Pathology \& Laboratory Medicine, 135(1), 135-142. https://doi. org/10.1043/2009-0698-OAR1.1

Ke, F. (2008). A case study of computer gaming for math: Engaged learning from gameplay? Computers \& Education, 51(4), 1609-1620. https://doi.org/10.1016/j. compedu.2008.03.003

Ke, F. (2016). Designing and integrating purposeful learning in game play: A systematic review. Educational Technology Research and Development, 64(2), 219244. https://doi.org/10.1007/s11423-015-9418-1

Kishimoto, T. M., Pinazza, M. A., Morgado, R. F. C., \& Toyofuki, K. R. (2011). Jogo e letramento: Crianças de 6 anos no ensino fundamental. Educação e Pesquisa, 37(1), 191-210.

Klisch, Y., Miller, L. M., Wang, S., \& Epstein, J. (2012). The impact of a science education game on students' learning and perception of inhalants as body pollutants. Journal of Science Education and Technology, 21(2), 295-303. https://doi. org/10.1007/s10956-011-9319-y

Kosmas, P., loannou, A., \& Zaphiris, P. (2018). Implementing embodied learning in the classroom: Effects on children's memory and language skills. Educational Media International, 56(1), 59-74. https://doi.org/10.1080/09523987.201 8.1547948

Liberati, A., Altman, D. G., Tetzlaff, J., Mulrow, C., Gøtzsche, P. C., Ioannidis, J. P. A., ... Moher, D. (2009). The PRISMA statement for reporting systematic reviews and meta-analyses of studies that evaluate health care interventions: Explanation and elaboration. PLoS Medicine, 6(7), e1000100. https://doi.org/10.1371/journal. pmed.1000100

McCarroll, M. L., Pohle-Krauza, R. J., \& Martin, J. L. (2009). Active learning in the classroom: A muscle identification game in a kinesiology course. Advances in Physiology Education, 33(4), 319-322. https://doi.org/10.1152/advan.00013.2009 
McGonigal, J. (2012). A realidade em jogo: Por que os games nos tornam melhores e como eles podem mudar o mundo (1. ${ }^{a}$ ed.). Best Seller.

Michaelsen, L. K., \& Sweet, M. (2011). Team-based learning. New Directions for Teaching and Learning, 2011(128), 41-51. https://doi.org/10.1002/tl.467

Ministério da Educação. (2015). Base nacional comum curricular: Primeira versão. Ministério da Educação. Recuperado de http://basenacionalcomum.mec.gov. br/images/BNCC_El_EF_110518_versaofinal_site.pdf

Morford, Z. H., Witts, B. N., Killingsworth, K. J., \& Alavosius, M. P. (2014). Gamification: The intersection between behavior analysis and game design technologies. The Behavior Analyst, 37(1), 25-40. https://doi.org/10.1007/ s40614-014-0006-1

Neves, V. F. A., Castanheira, M. L., \& Gouvêa, M. C. S. (2015). O letramento e o brincar em processos de socialização na educação infantil: Brincadeiras diferentes. Revista Brasileira de Educação, 20(60), 215-244. https://doi.org/10.1590/ S1413-24782015206011

Perkoski, I. R., \& Souza, S. R. (2015). “O espião”: Uma perspectiva analítico comportamental do desenvolvimento de jogos educativos de tabuleiro. Perspectivas em Análise do Comportamento, 6(2), 74-88. https://doi.org/10.18761/pac.2015.020

Prince, M. (2004). Does active learning work? A review of the research. Journal of Engineering Education, 93(3), 223-231. https://doi. org/10.1002/j.2168-9830.2004.tb00809.x

Rico, M., Agudo, J. E., \& Sánchez, H. (2015). Language learning through handheld gaming: A case study of an english course with engineering students. Journal of Universal Computer Science, 21(10), 1362-1378.

Roesch, S., Jung, S., Huber, S., Artemenko, C., Bahnmueller, J., Heller, J., ... Moeller, K. (2016). Training arithmetic and orthography on a web-based and socially-interactive learning platform. International Journal of Education and Information Technologies, 10, 204-217.

Silva, M. C. F. R. (2014). A prática educativa baseada em evidências: Contribuições na formação de docentes interdisciplinares. Interdisciplinaridade, 1(5), 25-30.

Stolaki, A., \& Economides, A. A. (2018). The Creativity Challenge Game: An educational intervention for creativity enhancement with the integration of Information and Communication Technologies (ICTs). Computers \& Education, 123(July 2017), 195-211. https://doi.org/10.1016/j.compedu.2018.05.009 (retiraria "(July 2017)")

Thomas, G., \& Pring, R. (Eds.). (2007). Educação baseada em evidências: A utilização dos achados científicos para a qualificação da prática pedagógica. Artmed.

Tüzün, H., Yilmaz-Soylu, M., Karakuş, T., Inal, Y., \& Kizilkaya, G. (2009). The effects of computer games on primary school students' achievement and motivation in geography learning. Computers \& Education, 52(1), 68-77. https://doi.org/10.1016/j.compedu.2008.06.008

Yang, Y.T. C. (2012). Building virtual cities, inspiring intelligent citizens: Digital games for developing students' problem solving and learning motivation. Computers \&Education, 59(2), 365-377. https://doi.org/10.1016/j. compedu.2012.01.012

Yang, Y.T. C. (2015). Virtual CEOs: A blended approach to digital gaming for enhancing higher order thinking and academic achievement among vocational 
high school students. Computers \& Education, 81, 281-295. https://doi.org/10.1016/j. compedu.2014.10.004

Yen-Hua, C., Chiu-Pin, L., Chee-Kit, L., Yin-juan, S., \& Tak-Wai, C. (2012). A collaborative cross number puzzle game to enhance elementary students' arithmetic skills. The Turkish Online Journal of Educational Technology, 11(2), 1-14.

Yien, J., Hung, C., Hwang, G., \& Lin, Y. (2011). A game-based learning approach to improving students' learning achievements in a nutrition course. The Turkish Online Journal of Educational Technology, 10(2), 1-10.

Informação dos autores:

i Universidade Estadual de Londrina, Departamento de Psicologia Geral e Análise do Comportamento, Brasil. https: //orcid.org/0000-0002-4529-0959

ii Universidade Federal do Pará, Núcleo de Teoria e Pesquisa do Comportamento, Brasil. https://orcid.org/0000-0001-5427-8743

iii Universidade de Brasília, Instituto de Psicologia, Brasil. https://orcid.org/0000-0001-8544-4468

iv Universidade Estadual de Londrina, Departamento de Psicologia Geral e Análise do Comportamento, Brasil.

v Universidade Federal do Pará, Núcleo de Teoria e Pesquisa do Comportamento, Brasil.

Toda a correspondência relativa a este artigo deve ser enviada para:

Myenne Mieko Ayres Tsutsumi

Departamento de Psicologia Geral e Análise do Comportamento Universidade Estadual de Londrina (Brasil)

Rua Pedro Marcos Prado, 345, Bloco 07 Apto 11, Londrina, Paraná, Brasil

myennetsutsumi@gmail.com

Recebido em 21 de dezembro de 2019

Aceite para publicação em 1 de maio de 2020 


\title{
Evaluation of educational games in the teaching of academic content: A systematic literature review
}

\begin{abstract}
This study aimed to make a systematic literature review of empirical studies that evaluated the effect of educational games on learning academic subjects comparatively analyzing the effects of studies with different research designs. In the review, we included empirical articles and case reports that evaluated games as an intervention method for teaching formal school content, that mentioned or contained previously defined keywords and were published in English or Portuguese from January 2006 to September 2019. Initially, 171 records were localized, and 24 were selected based on the inclusion criteria. It was found that: children were the main participants; electronic games were the most used; and mathematics was the discipline used in most studies. In 13 studies group design was employed and it was found that the experimental group showed better academic performance when compared to the control group. In all 11 studies that used a single-case design, the post-test performance was greater than that of the pre-test. These results show that games affected increasing students' academic performance.
\end{abstract}

Keywords: Educational games; Teaching-learning;

Evidence-based education 


\section{Evaluación de juegos educativos en la enseñanza de contenidos} académicos: Una revisión sistemática de la literatura

\section{RESUMEN}

Este estudio tuvo como objetivo hacer una revisión sistemática de la literatura de estudios empíricos que evaluaran el efecto de los juegos educativos en el aprendizaje de temas académicos, comparando los efectos de los estudios con diferentes diseños de investigación. La revisión incluyó artículos empíricos e informes de casos que evaluaban los juegos como un método de intervención para enseñar contenido escolar formal, que mencionaban o contenían palabras clave previamente definidas y se publicaron en inglés o portugués desde enero de 2006 hasta septiembre de 2019. Inicialmente, se localizaron 171 registros y se seleccionaron 24 en función de los criterios de inclusión. Se encontró que: los niños fueron los principales participantes; los juegos electrónicos fueron los más utilizados; y las matemáticas fueron la disciplina utilizada en la mayoría de los estudios. En 13 estudios, se empleó el diseño del grupo y se encontró que el grupo experimental mostró un mejor rendimiento académico en comparación con el grupo de control. En los 11 estudios que utilizaron diseño de caso único, el rendimiento posterior a la prueba fue mayor que el rendimiento anterior. Estos resultados mostraron que los juegos tuvieron un efecto en el aumento del rendimiento académico de los estudiantes.

Palabras clave: Juegos educativos; Enseñanzaaprendizaje; Educación basada en evidencia 